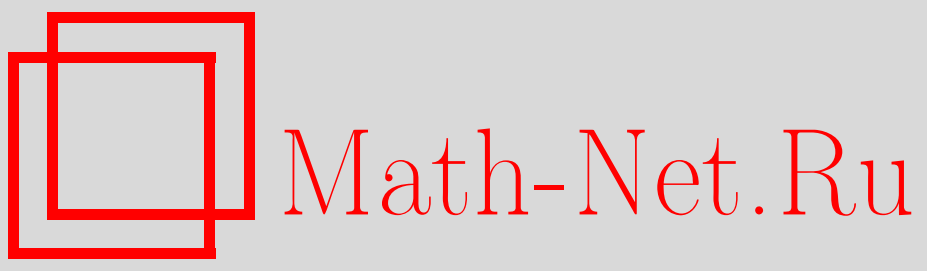

А. И. Штерн, Структурные свойства и ограниченные вещественные непрерывные 2-когомологии локально компактных групп, Функи. анализ и его прил., 2001, том 35, выпуск 4, 67-80

DOI: https://doi.org/10.4213/faa274

Использование Общероссийского математического портала MathNet.Ru подразумевает, что вы прочитали и согласны с пользовательским соглашением

http://www . mathnet.ru/rus/agreement

Параметры загрузки:

IP: 54.81 .137 .203

26 апреля 2023 г., 16:42:00

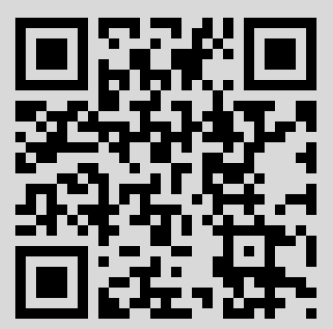


Функциональный анализ и его приложения

2001, т. 35, вып. 4, с. $67-80$

УДК 517.986.6

\title{
Структурные свойства и ограниченные вещественные непрерывные 2-когомологии локально компактных групп*
}

\author{
(C) 2001. А. И. ШТЕРН
}

\section{§1. Введение}

Вещественная функция $f$ на группе $G$ называется (вещественным) квазихарактером на этой группе, если множество $\{f(g h)-f(g)-f(h) \mid g, h \in G\}$ ограничено, и квазихарактер $f$ называется псевдохарактером, если $f\left(g^{n}\right)=n f(g)$ для любых $g \in G$ и $n \in \mathbb{Z}$. Теория псевдохарактеров, развитие которой началось в 1983 г. [5], оказалась глубоко связанной с теорией группы двумерных ограниченных когомологий с вещественными коэффициентами для дискретных групп [7].

Это соответствие между пространствами псевдохарактеров и когомологиями может быть следующим образом продолжено до соответствия между введенными Громовым [8] непрерывными ограниченными когомологиями топологических групп - точнее, группой двумерных ограниченных вещественных непрерывных когомологий $\widehat{H}^{2}(G)$ локально компактной группы $G-$ и пространством непрерывных вещественных псевдохарактеров на этой группе.

Пусть $G$ - локально компактная группа, и пусть $H^{n}(G)$ есть $n$-я группа когомологий комплекса

$$
0 \longrightarrow \mathbb{R} \stackrel{d^{0}=0}{\longrightarrow} C^{1}(G) \stackrel{d^{1}}{\longrightarrow} C^{2}(G) \longrightarrow \cdots,
$$

где $C^{n}(G)$ - вещественное векторное пространство всех непрерывных вещественных функций $f$ на группе $G^{n}, f:\left(g_{1}, \ldots, g_{n}\right) \mapsto f\left(g_{1}, \ldots, g_{n}\right) \in \mathbb{R}, g_{i} \in G$, а

$$
\begin{aligned}
d^{n} f\left(g_{1}, \ldots, g_{n}\right)=f\left(g_{2}, \ldots, g_{n}\right)+\sum_{i=1}^{n}(-1)^{i} f\left(g_{1}, \ldots, g_{i} g_{i+1}, \ldots, g_{n+1}\right) & \\
& +(-1)^{n+1} f\left(g_{1}, \ldots, g_{n}\right)
\end{aligned}
$$

(см. [1]; ср. также [2], где эти группы называются ванэстовскими группами вещественных когомологий, соответствующими тривиальному действию группы $G$ на $\mathbb{R})$. Группы $\widehat{H}^{n}(G)$ ограниченных вещественных непрерывных когомологий группы $G$ определяются аналогично с помощью формул (1) и (2), в которых пространства $C^{n}(G)$ заменены пространствами $C B^{n}(G)$ ограниченных непрерывных функций на $G^{n}$. В настоящей статье изучается группа $\widehat{H}^{2}(G)$.

Естественное отображение $\widehat{H}^{n}(G) \rightarrow H^{n}(G)$ определяется рассмотрением ограниченных непрерывных коциклов как непрерывных коциклов без условия

* Работа частично поддержана Российским фондом фундаментальных исследований (проект 9901-01211). 
ограниченности цепей. Упомянутая выше связь между группой двумерных ограниченных вещественных непрерывных когомологий $\widehat{H}^{2}(G)$ локально компактной группы $G$ и пространством непрерывных вещественных псевдохарактеров на этой группе состоит в том, что при $n=2$ ядро $\widetilde{H}^{2}(G)$ отображения $\widehat{H}^{2}(G) \rightarrow H^{2}(G)$ естественно изоморфно (векторному) факторпространству $Q P(G)$ пространства $P(G)$ всех непрерывных псевдохарактеров на $G$ по векторному подпространству (обычных) непрерывных (вещественных) характеров группы $G$; см. [17-19].

Особую роль в строении группы $\widehat{H}^{2}(G)$ играет компонента единицы данной локально компактной группы $G$. Этот феномен имеет место и для хорошо изученных ванэстовских групп $H^{n}(G)$ (см. $\left.[1,2]\right)$, поскольку группа $H^{n}(G)$ естественно изоморфна группе $H^{n}(G / K)$, где $K$ - такой компактный нормальный делитель в $G$, что компонента единицы факторгруппы $G / K$ является группой Ли (ср. лемму 3 ниже; аналогичное рассуждение позволяет доказать, что группа $H^{n}(G)$ конечномерна для любой почти связной группы $G$, ср. замечание 4$)$. Строение группы $\widehat{H}^{2}(G)$ определяется ее образом в $H^{2}(G)$ и упомянутым выше ядром $\widetilde{H}(G)$ отображения $\widehat{H}^{2}(G) \rightarrow H^{2}(G)$, которое может быть описано с помощью псевдохарактеров на $G$.

В настоящей статье с помощью некоторых вспомогательных результатов о структуре локально компактных групп для данной локально компактной группы $G$ строится такой «достаточно большой» нормальный делитель $N$ в $G$, что компонента единицы в группе $G / N$ является группой Ли, причем векторные пространства $\widehat{H}^{2}(G)$ и $\widehat{H}^{2}(G / N)$ естественно изоморфны (см. предложения 1 и 2). Эти факты вместе с вспомогательной теоремой о существовании сечений (теорема 1) используются в описании пространств $Q P(G)$ и $\widehat{H}^{2}(G)$ для любой связной локально компактной группы $G$ и в доказательстве конечномерности пространства $Q P(G)$ (факторпространства нетривиальных непрерывных вещественных псевдохарактеров по подпространству обычных характеров) и пространства $\widehat{H}^{2}(G)$ для любой почти связной локально компактной группы $G$. Основными результатами являются теоремы 2-7.

Часть результатов статьи опубликована без доказательств в [19].

\section{§2. Структура локально компактных групп: наибольший компактный нормальный делитель в группе и в ее компоненте единицы}

Обозначим через $G_{0}$ связную компоненту единицы локально компактной группы $G$.

ЛЕмма 1. Пусть $G$ - локально компактная группа, и пусть $K\left(G_{0}\right)-$ объединение всех компактных нормальных делителей группь $G_{0}$. Тогда $K\left(G_{0}\right)$ является наибольшим компактным нормальным делителем группь $G_{0}(m . e$. $K\left(G_{0}\right)$ содержит любой компактный нормальный делитель группь $\left.G_{0}\right)$ u ее характеристической подгруппой; в частности, $K\left(G_{0}\right)$ - компактный нормальный делитель в $G$. Связная компонента единицьь факторгруппь $G / K\left(G_{0}\right)$ является группой Ли.

ДоКАЗАТЕЛьствО. Рассмотрим наибольший компактный нормальный делитель $K\left(G_{0}\right)$ связной компоненты единицы $G_{0}$ данной локально компактной группы $G$, построенный Ивасавой в [10, Lemma 4.2]; там доказано также, что $G_{0} / K\left(G_{0}\right)$ 
есть группа Ли. Любой автоморфизм группы $G$ оставляет инвариантной компоненту $G_{0}$ и переводит любой компактный нормальный делитель в $G_{0}$ в некоторый компактный нормальный делитель в $G_{0}$ и, следовательно, в $K\left(G_{0}\right)$; поэтому $K\left(G_{0}\right)$ - характеристическая подгруппа в $G_{0}$ и, в частности, нормальный делитель в $G$.

Утверждения леммы 1 достаточно для доказательства предложения 1, но в доказательстве теоремы 7 нужен наибольший компактный нормальный делитель в почти связной группе (напомним, что локально компактная группа называется почти связной, если факторгруппа $G / G_{0}$ компактна). Лемма 2 дает простые достаточные условия существования наибольшего компактного нормального делителя в локально компактной группе. Ниже в замечании 1 мы обсудим соотношение между используемыми нами соображениями и результатами статьи [21].

ЛЕмма 2. Локально компактная группа имеет наибольиий компактный нормальный делитель $K(G)$ (который содержит любой компактньий нормальный делитель данной группь и поэтому является ее характеристической подгруппой), если факторгруппа $G / G_{0}$ имеет наибольший компактный нормальный делитель. Если $G$ - почти связная локально компактная группа, то факторгруппа $G / K(G)$ является группой Ли с конечным числом связных компонент.

ЗАмЕЧАНИЕ 1. В [21] Терп изучал ICS-свойство локально компактных групп, которое состоит в том, что любая возрастающая цепь компактных подгрупп данной локально компактной группы содержится в некоторой компактной подгруппе этой группы. Очевидно, что любая локально компактная ICS-группа $G$ содержит наибольший компактный нормальный делитель $K$ (это означает, что любой компактный нормальный делитель в $G$ содержится в $K)$. В теореме 2 из [21] утверждается, что локально компактная группа $G$ обладает ICS-свойством тогда и только тогда, когда группа $G / G_{0}$ обладает этим свойством. Лемма 2 может рассматриваться как частный случай этого утверждения. Однако доказательство теоремы 2 в [21] основано лишь на использовании результатов статьи [11], в которой доказано, что в любой почти связной локально компактной группе $H$ существует компактная подгруппа, образ которой в $H / H_{0}$ есть вся группа $H / H_{0}$, и при этом в доказательстве теоремы 2 в [21] не предпринимается никакой попытки доказать, что для любой возрастающей цепи $K_{\alpha}$ компактных подгрупп группы $G / G_{0}$ мы действительно можем сохранить монотонность, т. е. выбрать возрастающую цепь соответствующих компактных подгрупп в $H$ с заданными растущими компактными образами в $H / H_{0}$. Этот пробел, не допускающий очевидного восполнения, не позволяет использовать теорему Терпа.

Условие леммы 2 является достаточным, но не необходимым для существования наибольшего компактного нормального делителя. Например, в алгебраической подгруппе группы движений плоскости, определяемой дискретной подгруппой группы вращений, отвечающей множеству углов поворота, соизмеримых с $\pi$, и снабженной обычной топологией плоскости на сдвигах и дискретной топологией на вращениях (так что группа оказывается расширением дискретной группы корней из единицы, рассматриваемых как вращения обычной плоскости, с помощью группы сдвигов этой плоскости), имеется очевидный (единичный) наибольший компактный нормальный делитель, но факторгруппа по компоненте единицы является объединением конечных групп. 
Отметим также, что компонента единицы наибольшего компактного нормального делителя (если он существует) совпадает с наибольшим связным нормальным делителем Ивасавы [10, Theorem 14].

ДОКАЗАТЕЛЬСТвО ЛЕммы 2. Если $G$ почти связна, то $G$ проективно-лиева, и лиев фактор $L_{1}=G / N_{1}$ по компактному нормальному делителю $K_{1}$ имеет наибольший компактный нормальный делитель $M_{1}$, полный прообраз которого $K_{1} \supset N_{1}$ есть компактный нормальный делитель в $G$. Если $K_{2}$ - тоже компактный нормальный делитель в $G$, то $K_{1} K_{2}$ - компактный нормальный делитель, образ которого в $L_{1}$ есть компактный нормальный делитель $M$; поэтому $M \subset M_{1}$ и $K_{1} K_{2} \subset K$, т. е. $K_{1}$ - наибольший компактный нормальный делитель в $G$. В общем случае, пусть $K_{0}-$ наибольший компактный нормальный делитель в $G / G_{0}$. Прообраз $H$ группы $K_{0}$ в $G$ почти связен, и поэтому $H$ имеет наибольший компактный нормальный делитель $K$, который является характеристической подгруппой и, следовательно, нормальным делителем в $G$. С другой стороны, поскольку образ в $G / G_{0}$ любого компактного нормального делителя $K_{1}$ группы $G$ является компактным нормальным делителем в $G / G_{0}$, то этот образ содержится в $K_{0}$. Следовательно, $K_{1} \subset H$. Будучи нормальным делителем в $G, K_{1}$ является нормальным делителем и в $H$. Таким образом, $K_{1} \subset K$. Итак, любой компактный нормальный делитель группы $G$ содержится в компакте $K$. Следовательно, объединение $N$ всех компактных нормальных делителей в $G$ содержится в $K$, как и замыкание $\bar{N}$ этого объединения, и этот компактный нормальный делитель $\bar{N}$ группы $G$ является наибольшим по построению.

\section{§ 3. Структура локально компактных групп: наибольший аменабельный нормальный делитель, принадлежащий компоненте единицы}

Обратимся теперь к изучению «аменабельного радикала» локально компактной группы.

ПрЕДЛОЖЕНИЕ 1. Пусть $G$ - локально компактная группа, u пусть $A\left(G_{0}\right)$ - объединение всех аменабельных нормальных делителей группь $G_{0}$. Тогда $A\left(G_{0}\right)$ - наибольший аменабельный нормальный делитель группь $G_{0}$ (m. е. он содержит любой аменабельный нормальный делитель в $\left.G_{0}\right)$, он замкнут и является характеристической подгруппой; в частности, $A\left(G_{0}\right)$ - замкнутый аменабельный нормальный делитель в $G$. Кроме того, $G_{0} / A\left(G_{0}\right)$ - связная полупростая группа Ли без центра и без компактных простых сомножителей.

ДокАЗАТЕЛьство. Существование наибольшего аменабельного нормального делителя в любой локально компактной группе известно [13, Problem (0.26)]. Kроме того, в [10, Lemma 5.2] для любой связной локально компактной группы $H$ построен наибольший нормальный делитель $P$, компонента единицы которого есть проективный предел расширений компактных групп Ли с помощью связных разрешимых групп Ли (таким образом, $P$ есть расширение дискретной абелевой группы с помощью связного аменабельного нормального делителя), а факторгруппа $H / P$ - прямое произведение присоединенных групп простых некомпактных групп Ли. Таким образом, $P$ и есть наибольший аменабельный нормальный делитель в $G$. Наконец, из определения наибольшего аменабельного нормального делителя сразу следует, что $A\left(G_{0}\right)$ - характеристическая подгруппа в $G_{0}$, и это завершает доказательство предложения 1. 
Легко видеть, что (обычный) радикал «аменабельного радикала» $A\left(G_{0}\right)$ совпадает с радикалом $R(G)$ группы $G$; таким образом, из [13] и [4, теорема $(7.14)]$ следует, что компонента $\left(A\left(G_{0}\right)\right)_{0}$, которая связна и аменабельна, есть расширение наибольшего связного компактного нормального делителя [10, Theorem 14] факторгруппы $G / R(G)$ с помощью радикала $R(G)$. Кроме того, факторгруппа $A\left(G_{0}\right) /\left(A\left(G_{0}\right)\right)_{0}$ есть (возможно, единичная) конечно порожденная дискретная абелева группа (напомним, что вполне несвязный нормальный делитель в связной группе централен, а замкнутый вполне несвязный нормальный делитель в группе Ли конечно порожден и дискретен).

\section{§4. Редукция псевдохарактеров на локально компактной группе}

ПрЕДЛОЖЕНИЕ 2 (ср. [20]). Пусть $G$ - локально компактная группа, $A\left(G_{0}\right)$ - наибольший аменабельный нормальный делитель в $G_{0}$ (см. предложение 1), $B=\left[A\left(G_{0}\right), A\left(G_{0}\right)\right]$ - замыкание коммутаторной подгруппь группь $A\left(G_{0}\right) u$ $K\left(G_{0}\right)$ - наибольший компактньй нормальный делитель в $G_{0}$. Пусть $Q\left(G_{0}\right)=$ $K\left(G_{0}\right) B$ (замкнутая подгруппа, порожденная произведениями элементов из $K\left(G_{0}\right)$ и $\left.B\right)$. Тогда $Q=Q\left(G_{0}\right)$ - характеристическая подгруппа в $G, G_{0} / Q-$ расширение полупростой группь Ли без цүентра и без компактных факторов с помощью абелевой группь Ли и любой непрерывный псевдохарактер на $G$ обращается в нуль на $Q$ (так что он определяется непрерывным псевдохарактером на $G / Q[6])$.

ДоКАЗАТЕЛЬСтвО. Любой непрерывный псевдохарактер $\varphi$ на $G$ обращается в нуль на наибольшем компактном нормальном делителе $K\left(G_{0}\right) \subset G_{0}$, потому что любой ограниченный псевдохарактер тождественно равен нулю. Следовательно, псевдохарактер $\varphi$ определяется непрерывным псевдохарактером $\Phi$ на $H=G / K\left(G_{0}\right)[6]$. По лемме 1 ограничение псевдохарактера $\Phi$ на аменабельную группу $A\left(H_{0}\right)$ является обычным характером этой группы. Так как $K\left(G_{0}\right) \subset G_{0}$, то образ группы $G_{0}$ есть $H_{0}$ (см. $[9$, Corollary 7.13$\left.]\right)$, и поэтому образ группы $A\left(G_{0}\right)$ принадлежит $A\left(H_{0}\right)$ (как замкнутый аменабельный нормальный делитель в $\left.H_{0}\right)$. Поэтому ограничение псевдохарактера $\varphi$ на $A\left(G_{0}\right)$ есть обычный характер группы $A\left(G_{0}\right)$, откуда следует, что $\varphi$ обращается в нуль на $\left[A\left(G_{0}\right), A\left(G_{0}\right)\right]$ (и, конечно, на $\left.K\left(G_{0}\right)\right)$. Таким образом, $\left.\varphi\right|_{Q}=0$. Кроме того, $Q-$ характеристическая подгруппа, потому что $A\left(G_{0}\right)$ и $K\left(G_{0}\right)$ - характеристические подгруппы. Наконец, группа $(G / Q)_{0}$ есть замыкание канонического образа группы $G_{0}$, но этот образ есть факторгруппа группы Ли $G_{0} / K\left(G_{0}\right)$, откуда следует, что $(G / Q)_{0}$ - не что иное, как канонический образ группы $G_{0} / K\left(G_{0}\right)$. Этот образ есть расширение группы $G_{0} / A\left(G_{0}\right)$ с помощью $A\left(G_{0}\right) / Q$, где $G_{0} / A\left(G_{0}\right)$ полупроста и не имеет компактных сомножителей, а $A\left(G_{0}\right) / Q$ абелева. Это завершает доказательство предложения 2 .

ЗАмЕЧАНИЕ 2. Вообще говоря, группа $Q=Q\left(G_{0}\right)$ (см. предложение 2) не является наибольшим нормальным делителем $N$ в данной локально компактной группе $G$, для которого пространства псевдохарактеров на $G$ и $G / N$ естественно изоморфны. Бо́льший нормальный делитель с этим же свойством указан в [20].

ЗАмЕЧАНИЕ 3. Из доказательства предложения 2 следует, что среди полупростых факторгрупп данной связной локально компактной группы $G$, не имеющих связных компактных нормальных делителей, имеется наибольшая (т.е. такая, что любая факторгруппа рассматриваемого класса есть образ этой факторгруппы 
при естественной факторизации), и этой наибольшей группой является факторгруппа $S(G)=G / A(G)_{0}$ по компоненте единицы $A(G)_{0}$ нормального делителя $A(G)$, причем, как мы видели, $A(G)_{0}$ есть расширение наибольшего связного компактного нормального делителя $K(G / R)_{0}$ с помощью радикала $R=R(G)$.

\section{§5. Когомологии и ограниченные когомологии: переход к группам Ли}

Напомним, что для локально компактной группы $G$ обозначение $K\left(G_{0}\right)$ введено в лемме 1.

ЛЕмма 3. Пусть $G$ - локально компактная группа, а $f$ - вещественный непрерывный 2-коцикл на G. Этот коцикл когомологичен некоторому вещзественному непрерьвному 2-коциклу g на $G$, постоянному на сменных классах по нормальному делителю $K\left(G_{0}\right)$. Если коциикл $f$ ограничен, то $g$ тоже ограничен.

ДОКАЗАТЕЛЬСТВО. Если

$$
f(g, h)=f(h, k)-f(g h, k)+f(g, h k), \quad g, h, k \in G,
$$

где $f$ непрерывен, то, интегрируя по переменной $k \in K\left(G_{0}\right)$ относительно меры Хаара на $K\left(G_{0}\right)$, мы получаем

$$
f(g, h)=F(h, e)-F(g h, e)+F(g, h), \quad g, h \in G,
$$

где

$$
F(g, h)=\int_{K(G)} f(g, h k) d \mu(k), \quad g, h \in G .
$$

Из соотношения (3) следует, что коцикл $f$ когомологичен функции $\Phi:(g, h) \mapsto$ $F(g, h)-F(g, e), g, h \in G$, которая $K\left(G_{0}\right)$-инвариантна по $g \in G$ и ограничена, если $f$ ограничена.

Интегрируя соотношение

$$
\Phi(h, k)=\Phi(g, h)-\Phi(g, h k)+\Phi(g h, k), \quad h, k \in G, g \in K\left(G_{0}\right),
$$

по $g \in K\left(G_{0}\right)$ и применяя те же рассуждения, что и выше, мы получаем искомую когомологическую эквивалентность данного коцикла двусторонне $K\left(G_{0}\right)$-инвариантому коциклу, который ограничен, если исходный коцикл $f$ ограничен.

Приведенное выше доказательство леммы 3 использует универсальную идею усреднения. Для группы обычных когомологий в частном случае групп Ли утверждение можно извлечь также из [1, гл. 3, следствие 2.2].

ЗАмЕЧАНИЕ 4. Из леммы 3 следует, что векторные пространства $H^{n}(G)$ и $H^{n}\left(G / K\left(G_{0}\right)\right)$ изоморфны и векторные пространства $\widehat{H}^{n}(G)$ и $\widehat{H}^{n}(G / N)$ также изоморфны. Если $G$ связна, то $G / K\left(G_{0}\right)$ - группа Ли и, следовательно, $H^{n}\left(G / K\left(G_{0}\right)\right)$ конечномерна, а поэтому и $H^{n}(G)$ конечномерна для любого $n \in \mathbb{N}$.

\section{§6. Соответствие между ограниченными непрерывными коциклами на локально компактной группе $G$ и псевдохарактерами на одномерных центральных расширениях этой группы}

Пусть $H$ - центральное расширение локально компактной группы $G$ с помощью группы $\mathbb{R}$, и пусть $\pi: H \rightarrow G-$ каноническое отображение. Как известно, непрерывное сечение $s: G \rightarrow H$ (являющееся правым обратным к $\pi$ ) существует тогда и только тогда, когда это расширение определяется непрерывным коциклом. 
В дальнейшем нам будет полезен следующий факт, гарантирующий существование (глобальных) непрерывных сечений для одномерных центральных расширений локально компактных групп (этот результат был неявно использован в [18]).

Теорема 1. Любое топологическое расширение локально компактной группь $G$ с помощью односвязной группы Ли допускает глобальное непрерывное сечение.

ДокАЗАТЕльство. Существование локального непрерывного сечения следует из теоремы Серра [16] (доказательство приведено также в [12]; отметим, что сам Сepp в [15] приписал эту теорему Глисону со слов Бурбаки, но в работах Глисона был опубликован только случай действия компактной группы Ли). Ввиду односвязности нормального делителя существование глобального непрерывного сечения следует теперь из [14, Theorem (B.1)].

Теорема 1 означает, в частности, что любое расширение локально компактной группы с помощью векторной группы топологически тривиально. Таким образом, в рассматриваемом случае задачи, касающиеся соответствующих расширений групп, могут решаться именно на уровне ванэстовских групп когомологий и не требуют привлечения сигаловских групп [14], ср. [2, гл. 2, §2, теорема 4].

Соответствие между непрерывными псевдохарактерами (по модулю векторного подпространства обычных вещественных характеров) на центральных расширениях локально компактной группы $G$ с помощью группы $\mathbb{R}$, определенных ограниченными 2 -коциклами на $G$ (по модулю кограниц, порожденных ограниченными непрерывными функциями), и коциклами, определяющими эти псевдохарактеры, устанавливается следующим образом [17-19].

Теорема 2. Пусть $G$ - локально компактная группа. Если $\psi$ - ограниченнацй непрерывный 2-коцикл на $G$, то формула

$$
\left(g_{1}, r_{1}\right)\left(g_{2}, r_{2}\right)=\left(g_{1} g_{2}, r_{1}+r_{2}+\psi\left(g_{1}, g_{2}\right)\right), \quad g_{1}, g_{2} \in G, r_{1}, r_{2} \in \mathbb{R},
$$

определяет центральное расширение $H$ группь $G$ с помощью $\mathbb{R} u$ отображение $f: H \rightarrow \mathbb{R}$, определяемое соотношением

$$
(g, r) \mapsto r, \quad(g, r) \in H,
$$

является (непрерывным) квазихарактером на Н. Соответствующий непрерывный псевдохарактер чна Н задается формулой

$$
\varphi(g, r)=r+\lim _{n \rightarrow \infty} \frac{1}{n} \sum_{k=1}^{n-1} \psi\left(g, g^{k}\right), \quad(g, r) \in H .
$$

Обратно, пусть s - непрерывное сечение канонического отображения $\pi$ : $H \rightarrow G$. Пусть $f$ - непрерывный вещественный псевдохарактер на $H, u$ пусть $\psi$ - вещественная непрерывная 2-коцепь на $S$, определенная формулой

$$
\psi(x, y)=f(s(x) s(y))-f(s(x))-f(s(y)), \quad x, y \in G .
$$

Тогда функция $\psi$ есть ограниченный коцикл, и псевдохарактер $f$ нетривиален (не является обычным характером) тогда и только тогда, когда коцикл $\varphi$ нетривиален (не гомологичен нулю).

ДокАЗАТЕЛЬСтво. Функция $\psi$, определяемая формулой (7), действительно является коциклом; доказательство, приведенное в [19], существенно использует свойства псевдохарактеров на некоторых подгруппах группы $H$. Утверждение о 
существовании и свойствах предела в (6) следует из формул (1) и (2) и общих свойств квазихарактеров и псевдохарактеров (см. например, [17]): для любого квазихарактера $f$ на группе $G$ предел $\varphi(s)=\lim _{n \rightarrow \infty} n^{-1} f\left(s^{n}\right)$ существует для любого $s \in G$ (более того, этот предел совпадает с общим значением всех инвариантных средних на $\mathbb{Z}$ на ограниченной функции $n \mapsto f((n+1) s)-f(n s)$, $n \in \mathbb{Z})$. Взаимную обратность отображений $(6)$ и $(7)$ достаточно проверить в частном случае, когда данное расширение $H$ реализовано в виде $(1)$ при специальном выборе сомножителей, а именно, с помощью топологического изоморфизма $H \ni h \mapsto(\pi(h), \varphi(h)) \in G \times \mathbb{R}$ (топологическое произведение) с умножением $h_{1} h_{2} \mapsto\left(\pi\left(h_{1}\right) \pi\left(h_{2}\right), \varphi\left(h_{1}\right)+\varphi\left(h_{2}\right)+\left(\varphi\left(h_{1} h_{2}\right)-\varphi\left(h_{1}\right)-\varphi\left(h_{2}\right)\right), h_{1}, h_{2} \in H\right.$, где $\varphi\left(h_{1} h_{2}\right)-\varphi\left(h_{1}\right)-\varphi\left(h_{2}\right)$ не меняется при изменении $h_{1}$ и $h_{2}$ на элементы группы $\mathbb{R}$; полученный 2 -коцикл на $G$ согласуется с формулой (7) при выборе сечения $s(g)=(g, 0) \in H, g \in G$. Наконец, утверждения об эквивалентности коциклов относительно добавления обычных характеров к псевдохарактерам сразу следуют из формулы (7), а утверждения об инвариантности получаемых псевдохарактеров относительно возмущений коциклов кограницами ограниченных цепей следуют из (6).

ЗАмЕЧАНИЕ 5. Как известно, одномерное центральное расширение $H=G \rtimes \mathbb{R}$ данной группы $G$, определяемое некоторым коциклом $\psi$, расщепимо (в полупрямое произведение) тогда и только тогда, когда $\psi$ является кограницей. Для ограниченного коцикла $\psi$ это условие выполняется тогда и только тогда, когда $\psi$ есть кограница некоторой вещественной непрерывной функции $f$ на группе $G$, которая, таким образом, является квазихарактером и поэтому ограниченно когомологична некоторому псевдохарактеру $\theta$ на $G$. Рассмотрим $\theta$ как псевдохарактер на $H$. Тогда непосредственное вычисление показывает, что условие расщепимости равносильно тому, что разность $f-\varphi$, где $\varphi$ определен формулой (6), есть кограница, определяемая ограниченной 1-коцепью (ограниченной вещественной непрерывной функцией на $H)$, или тому, что $\theta-\varphi$ есть обычный непрерывный вещественный характер. Таким образом, $\psi$-расширение расщепимо тогда и только тогда, когда $\varphi$ есть сумма непрерывного псевдохарактера на $H$, определяемого некоторым псевдохарактером на $G$, и некоторого обычного вещественного характера на $H$.

Приложения соответствия, описанного в теореме 2 , к вполне несвязным (и, в частности, дискретным) группам мы обсудим в другой работе.

\section{§7. Пространства псевдохарактеров, ограниченные когомологии и коциклы Гишарде-Вигнера}

Приведем сначала условия тривиальности для пространств $Q P(G)$. Напомним, что связная локально компактная группа $G$ есть проективный предел связных факторгрупп Ли (по компактным нормальным делителям), и, согласно теореме Леви-Мальцева, каждая из этих факторгрупп Ли есть расширение полупростой группы Ли с помощью разрешимой группы Ли. В свою очередь, каждая из полученных полупростых групп Ли есть расширение полупростой связной группы Ли $S$ без нетривиальных компактных связных нормальных делителей (с точностью до изоморфизма «стабильная» группа $S$ не зависит от выбора исходного «достаточно малого» компактного нормального делителя; в замечании 3 мы обозначили ее через $S(G))$ с помощью связной компактной полупростой группы Ли. 
Очевидно, любая полупростая факторгруппа Ли группы $G$ без нетривиальных компактных связных нормальных делителей есть факторгруппа группы $S(G)$, и в этом смысле группа $S(G)$ есть наибольшая полупростая некомпактная факторгруппа Ли группы $G$.

ПрЕДЛОЖЕНИЕ 3 [17-19]. Следующие условия эквивалентны для любой связной локально компактной группь $G$ :

(1) любой непрерывный псевдохарактер на $G$ является обычным вещественным характером $G$;

(2) центр наибольшей полупростой факторгруппь Ли $S(G)$ без нетривиальных компактных связных нормальных делителей конечен, и группа $G$ не имеет нетривиальной факторгруппь Ли, изоморфной одномерному вещзественному иентральному расширению группь $S$ с помощью $\mathbb{R}$, определенному ограниченным непрерывным вещественным 2-коциклом на $S$.

Мы дополняем это предложение описанием (см. теоремы 3-5 ниже) векторных пространств $Q P(G)$ и $\widehat{H}^{2}(G)$ для связной локально компактной группы $G$ в терминах структуры некомпактной части $T$ полупростой группы Ли, являющейся наибольшей полупростой факторгруппой без центра наибольшей полупростой факторгруппы Ли $S$ группы $G$.

Для связной группы Ли $H$ обозначим через $\widetilde{H}$ односвязную группу Ли, локально изоморфную $H$ (как известно, $\widetilde{H}$ определена однозначно с точностью до топологического изоморфизма). Для локально компактной группы $G$ мы будем в этом параграфе обозначать через $A$ группу $A\left(G_{0}\right)$, введенную в предложении 1 , и через $Q$ группу $Q\left(G_{0}\right)$, введенную в предложении 2. Следующая теорема 3 означает, что группа $\widehat{H}^{2}(G)$ двумерных вещественных непрерывных когомологий локально компактной группы $G$ естественно изоморфна соответствующей группе для полупростой факторгруппы $G / A$ (без центра и без компактных факторов), группа $Q P(G)$ - группе $Q P(G / Q)$, а группа $\widetilde{H}^{2}(G)$ - группе $\widetilde{H}^{2}(G / Q)$.

Теорема 3. В предьдущих обозначениях для любой локально компактной группь $G$ имеют место естественные изоморфизмы $\widehat{H}^{2}(G) \simeq \widehat{H}^{2}(G / A)$, $Q P(G) \simeq Q P(G / Q)$ и $\widetilde{H}^{2}(G) \simeq \widetilde{H}^{2}(G / Q)$, определяемье подбемом элементов групп, стоящих в правых частях әтих соотношений.

ДокАЗАТЕЛЬствО. Как следует из описания соответствия между элементами пространства $\widehat{H}^{2}(G)$ и элементами пространства $P(G \rtimes \mathbb{R})$, см. теорему 2 в $\$ 6$, любой непрерывный ограниченный коцикл на $G$ определяет одномерное центральное расширение $G \rtimes \mathbb{R}$ и непрерывный псевдохарактер на $G \rtimes \mathbb{R}$. Если полученный псевдохарактер на $G \rtimes \mathbb{R}$ тривиален на $\mathbb{R}$, то он определяется псевдохарактером на $G$, а значит, на $G / A$, а значит, и псевдохарактером на $(G / A) \rtimes \mathbb{R}$, тривиальным на $\mathbb{R}$, и поэтому определяет непрерывный ограниченный коцикл на $G / A$. Если же полученный псевдохарактер на $G \rtimes \mathbb{R}$ нетривиален на $\mathbb{R}$, то он определяется псевдохарактером на $M=G \rtimes \mathbb{R} / A_{G \rtimes \mathbb{R}}$, нетривиальным на образе группы $\mathbb{R}$ в этой факторгруппе. Следовательно, этот псевдохарактер на $M$ определяет псевдохарактер на одномерном центральном расширении группы $G / A$ и потому непрерывный ограниченный коцикл на самой группе $G / A$. Так как полученное соответствие между пространством $\widehat{H}^{2}(G)$ (классов ограниченно гомологичных непрерывных вещественных коциклов на $G$ ) и пространством $\widehat{H}^{2}(G / A)$ (классов ограниченно гомологичных коциклов на $G / A)$ взаимно однозначно и, очевидно, 
сохраняет выпуклые комбинации (см. формулы (6) и (7)), то оно является изоморфизмом линейных пространств. Это завершает доказательство утверждения об изоморфности пространств $\widehat{H}^{2}(G)$ и $\widehat{H}^{2}(G / A)$. Второе соотношение следует из предложения 2 , а третье равносильно второму, поскольку группа $\widetilde{H}^{2}(G)$ естественно изоморфна аддитивной группе $Q P(G)($ см. [7]).

Таким образом, вычисление группы $\widehat{H}^{2}(G)$ для данной связной локально компактной группы $G$ сводится к вычислению соответствующей группы $\widehat{H}^{2}$ для связной полупростой группы Ли $S$ без центра и без компактных полупростых факторов, а вычисление групп $\widetilde{H}^{2}(G)$ и $Q P(G)$ сводится к вычислению соответствующих групп для расширения $S \rtimes V$ связной полупростой группы Ли без центра и без компактных полупростых факторов с помощью векторной группы $V$. Перейдем к описанию группы $\widehat{H}^{2}(S)$.

Теорема 4. Пусть $S$ - связная полупростая (вещественная) группа Ли, пусть $T$ - простая группа Ли, являющаяся факторгруппой группь $S, u$ пусть изентр соответствующей односвязной группь Ли $\widetilde{T}$ бесконечен $(m$.е. ассоциированное с т симметрическое пространство является эрмитово симметрическим).

(1) Поднятие на $S$ коцикла Гишарде-Вигнера, связанного с присоединенной группой группь $\widetilde{T}([9]$, см. также [1, гл. III, предложение 7.6]), есть ограниченный вещественный непрерывный 2-коцикл на $S$.

(2) Любой ограниченный вещественный непрерывный 2-коичкл на $S$ когомологичен некоторой линейной комбинации поднятий коциклов ГишардеВигнера, отвечающих всевозможным присоединенным группам простых факторгрупп T, у которых цеентр универсальной накрывающей группь бесконечен.

(3) Размерность векторного пространства $\widehat{H}^{2}(S)$ равна числу некомпактных простых прямых сомножителей $T$ в группе $S$, для которых иентр группь $\widetilde{T}$ бесконечен.

Опишем еще одну связь между теоремами 3 и 4. Пусть $[H, H]$ - замыкание коммутаторной подгруппы радикала $H$ группы $G$ [13, Proposition 3.7], пусть $V$ - векторная часть связной абелевой факторгруппы $H /[H, H]$, пусть $K-$ наибольший компактный нормальный делитель группы $H /[H, H]$, и пусть $M-$ факторгруппа группы $G /[H, H]$ по нормальному делителю $K$. Тогда $M-$ центральное расширение полупростой группы Ли $S=G / H$ с помощью векторной группы $V$ и любой непрерывный псевдохарактер на $G$ допускает факторизацию СКвозь $M[17,18]$.

ДОКАЗАТЕЛЬСТВО ТЕОРЕМЫ 4. Пусть $T$ - ПроизвольНЫЙ Полупростой некомпактный фактор группы $S$, для которого центр соответствующей односвязной группы Ли бесконечен (и, таким образом, соответствующее симметрическое пространство является эрмитово симметрическим; список этих пространств приведен, например, в $[1,3,9])$, и пусть $K_{T}$ - аналитическая подгруппа группы $T$, отвечающая максимальной компактной подалгебре Ли алгебры Ли группы Ли $T$. Осуществим изоморфное отождествление центра $Z_{K_{T}}$ аналитической группы $K_{T}$ с полем вещественных чисел $\mathbb{R}$ (например, с помощью натурального параметра). Рассмотрим разложение Ивасавы $p=k(t) a n, t \in T$, связанное с группой $K_{T}$. Отображение $t \mapsto k(t)$, переводящее каждый элемент $t \in T$ в «компактную» 
компоненту $k(t) \in K_{T}$ его разложения, является непрерывным. Рассмотрим композицию отображения $t \mapsto k(t)$ и непрерывной проекции, отображающей каждый элемент $k \in K_{T}$ в его центральную составляющую $z(k) \in Z_{K_{T}}$. Эта композиция определяет некоторый квазихарактер на $T$. (По причинам, описанным ниже, мы будем называть псевдохарактер, соответствующий этому квазихарактеру, псевдохарактером Гишарде-Вигнера.) Доказательство этого факта, приведенное в [19], довольно громоздко (в отличие от почти очевидного модельного примера, связанного с группой $\operatorname{SL}(2, \mathbb{R})$, в котором ограниченность коцикла Гишарде-Вигнера следует из ограниченности аргумента логарифма непрерывной величины, принимающей значения в открытой правой полуплоскости; см. [9]). С другой стороны, доказательство в [19] одновременно устанавливает, что коциклы Гишарде-Вигнера ограничены на любой простой группе Ли, на которой они существуют, поскольку ограниченный вещественный коцикл, связанный с только что построенным квазихарактером, совпадает с коциклом Гишарде-Вигнера (что сразу следует из сравнения нашей конструкции с [9]). Это доказывает утверждение (1).

Кроме того, любой непрерывный псевдохарактер на связной локально компактной группе $G$ определяется непрерывным псевдохарактером на одномерном центральном расширении $H$ полупростой факторгруппы $S=G / A(G)$ и поэтому допускает подъем на универсальную накрывающую группу $\widetilde{H}$, изоморфную полупрямому, а в силу центральности расширения $H$ прямому произведению $\widetilde{S} \times \mathbb{R}$. Псевдохарактер на прямом произведении есть сумма псевдохарактеров на сомножителях, но псевдохарактер на $\mathbb{R}$ есть обычный характер, а ненулевых характеров на $\widetilde{S}$ нет, тогда как любой псевдохарактер на универсальной накрывающей группе $\widetilde{S}$ группы $S$ есть сумма своих ограничений на простые факторы в $\widetilde{S}$, т. е. сумма некоторых псевдохарактеров Гишарде-Вигнера на этих сомножителях. В частности, любой непрерывный псевдохарактер на $S$ является некоторой линейной комбинацией стандартных псевдохарактеров Гишарде-Вигнера (снова с точностью до прибавления обычного характера). Это доказывает утверждение (2), и отсюда сразу следует утверждение (3) о размерности вещественного векторного пространства $\widehat{H}^{2}(S)$, что завершает доказательство теоремы 4.

Опишем теперь ядро $\widetilde{H}^{2}(L)$ естественного отображения $\widehat{H}^{2}(L) \rightarrow \widetilde{H}^{2}(L)$ $($ см. $\$ 1)$ и изоморфную этому ядру группу $Q P(L)$ для расширения $L$ полупростой группы с помощью векторной группы.

ТеоремА 5. Пусть L - связная (вещественная) группа Ли, являющаяся расширением полупростой группь Ли $S$ с помощью векторной группь $V$ относительно непрерывного 2 -коцикла $\varphi$ на группе $S$ со значениями в $V$. Пространство $\widetilde{H}^{2}(L)$ образовано коциклами, для которых соответствующзее расширение группь L с помощью $\mathbb{R}$ расщепимо (см. замечание (5)); как векторное пространство $\tilde{H}^{2}(L)$ порождено классами эквивалентности поднятий всевозможных коииклов Гишарде-Вигнера на простых односвязных факторгруппах группь $S$, имеющих бесконечный иентр, и классами эквивалентности всевозможных вещественных коциклов вида $f=F \circ \varphi$, где $F$ пробегает пространство $V^{*}$, дуальное $\kappa V$. Пространство $Q P(L)$ порождено классами эквивалентности псевдохарактеров Гишарде-Вигнера на простых односвязных факторгруппах группь $S$, имеющих бесконечный иентр, 
и классами эквивалентности всевозможных псевдохарактеров $\psi$ на L, определяемых вещественными коциклами вида $f=F \circ \varphi$ по формуле (7), где $F$ пробегает пространство $V^{*}$, дуальное $\kappa V$.

ДокАЗАТЕЛЬство. Из замечания 5 следует, что ядро $\widetilde{H}^{2}(L)$ образовано коциклами, для которых соответствующее расширение группы $L$ с помощью $V$ расщепимо, т.е. определяется коциклом, связанным с псевдохарактером на $L$ (а не на его одномерном расширении). Переходя к пересечению ядер обычных характеров на $L$, мы вправе считать, что на $L$ нет нетривиальных характеров и $Q P(L)$ есть линейное пространство непрерывных псевдохарактеров на $L$. Если некоторый псевдохарактер на $L$ нетривиален на $V$, то его ограничение на $V$ есть некоторый линейный функционал $F$ на $V$ (элемент пространства $V^{*}$ ); этот псевдохарактер определяет коцикл $F \circ \varphi$ на $S$ по формуле $(7)$, который разлагается по коциклам Гишарде-Вигнера по теореме 4. Если же данный псевдохарактер равен нулю на $V$, то он определяется псевдохарактером на $S$, структура которого известна $[17,19]$, и также имеет указанный в теореме вид. Это завершает доказательство теоремы 5.

ЗАмЕчАНИЕ 6 . Пусть $D-$ такая дискретная подгруппа центра группы $\widetilde{S}$, что $\widetilde{S} / D$ изоморфна $S$. Подъем псевдохарактера на $S$ до псевдохарактера на $\widetilde{S}$ есть линейная комбинация псевдохарактеров Гишарде-Вигнера на простых односвязных факторгруппах группы $\widetilde{S}$, имеющих бесконечный центр, и ограничение этого псевдохарактера на подгруппу $D$ центра группы $\widetilde{S}$ есть нулевой характер. Обратно, если псевдохарактер на группе $\widetilde{S}$ обращается в нуль на $D$, то он определяет псевдохарактер на группе $S$. (Дискретная группа $D$ может считаться подгруппой конечно порожденной свободной абелевой группы, отвечающей прямому произведению центров конечного числа односвязных простых групп эрмитово симметрического типа, и поэтому $D-$ свободная группа, ранг которой не превосходит ранга центра группы $\widetilde{S}$.

Из теорем 3-5 следует, что единственными простыми факторгруппами Ли данной полупростой группы Ли, которые могут внести ненулевой вклад как в обычную группу двумерных непрерывных вещественных ванэстовских когомологий, так и в группу двумерных непрерывных вещественных ограниченных когомологий, являются факторгруппы, связанные с эрмитово симметрическими пространствами (см. [1, гл. III, П. 7.5] и [18]). Рассмотрим пример, связанный с простой группой этого типа и иллюстрирующий теоремы 4 и 5.

Пример. Если $T$ - простая группа Ли, имеющая конечный центр и связанная с эрмитово симметрическим пространством, то коцикл Гишарде-Вигнера на $T$ определяет нетривиальный элемент и в группе обычных двумерных непрерывных вещественных ванэстовских когомологий (эта группа одномерна, см. [1, гл. III, предложение 7.5]), и в группе двумерных непрерывных вещественных ограниченных когомологий, в то время как нетривиальных псевдохарактеров на этом сомножителе нет [17-19], и, следовательно, ядро $\widetilde{H}^{2}(T)$ отображения этих групп когомологий тривиально. Таким образом, в рассматриваемом случае вклады сомножителя $T$ в обе когомологические группы (и в обычную ванэстовскую, и в группу ограниченных когомологий) одномерны, а вклад в ядро $\widetilde{H}^{2}(T)$ тривиален. Если же центр группы $T$ бесконечен, так что $T=\widetilde{T}$, то коцикл Гишарде-Вигнера определяет нетривиальный псевдохарактер на $\widetilde{T}$ и этот псевдохарактер единствен 
с точностью до умножения на скаляр; следовательно, ядро $\widetilde{H}^{2}(T)$ естественного отображения $\widehat{H}^{2}(T) \rightarrow H^{2}(T)$ одномерно. В то же время из теоремы ЛевиМальцева следует, что $H^{2}(T)=0$ (оставаясь внутри когомологической техники, можно рассмотреть коммутаторную подгруппу $P=\left[K_{T}, K_{T}\right]$ аналитической группы $K_{T}$ и применить очевидный аналог предложения 7.5 из [1, гл. III] вместе с теоремой ван Эста [1, гл. III, следствие 7.2] не к максимальной компактной подгруппе $K_{T}$, а к группе $P$, откуда следует формула $H^{*}(G)=H^{*}(\mathfrak{t}, \mathfrak{p})$ для алгебр Ли $\mathfrak{t}$ и $\mathfrak{p}$ групп $T$ и $P$ соответственно, что приводит к той же теореме тривиальности). Следовательно, если центр бесконечен, то группа $H^{2}(T)$ тривиальна, а обе группы $\widehat{H}^{2}(T)$ и $\widetilde{H}^{2}(T)$ одномерны.

\section{§ 8. Конечномерность группы $\widehat{H}^{2}(G)$ для почти связной локально компактной группы $G$}

Теперь мы можем доказать теорему конечномерности для группы двумерных ограниченных вещественных непрерывных когомологий произвольной связной локально компактной группы. Этот результат усилен ниже, см. теорему 7.

Теорема 6. Пусть $G-$ связная локально компактная группа. Группа двумерных ограниченных вещзественных непрерывных когомологий $\widehat{H}^{2}(G)$ конечномерна.

ДокАЗАТЕЛьствО. Это утверждение сразу следует из теоремы 4 и леммы 3 в сочетании с теоремой конечномерности для ванэстовских когомологий групп Ли [1, следствие III, 7.3].

В заключение мы распространим теорему конечномерности на случай почти связных локально компактных групп. Сначала мы установим соответствующий вариант леммы 3. Напомним, что для почти связной локально компактной группы $G$ в лемме 2 построен наибольший компактный нормальный делитель $K(G)$ и факторгруппа $G / K(G)$ есть группа Ли с конечным числом связных компонент.

ЛЕмма 4. Пусть $G$ - почти связная локально компактная группа, а $f$ вещественный непрерывный 2-коцикл на G. Этот коицкл когомологичен некоторому вещзественному непрерьвному 2 -коциклу $g$ на $G$, постоянному на смежных классах по наибольшему компактному нормальному делителю $K(G)$. Если коцикл $f$ ограничен, то $g$ тоже ограничен.

ДокАЗАТЕЛьство. Рассуждение не отличается от доказательства леммы 3.

Теперь мы усилим теорему 6.

ТЕОРема 7. Для любой почти связной локально компактной группь $G$ группь когомологий $H^{2}(G)$ (в смысле ван Эста) и $\widehat{H}^{2}(G)$ конечномерны.

ДоКАЗАТЕЛЬСтвО. Из теоремы 3 следует, что векторное пространство $Q P\left(G_{0}\right)$ конечномерно. Тогда и векторное пространство $Q P(G)$ конечномерно. Действительно, если два непрерывных вещественных псевдохарактера на $G$ совпадают на $G_{0}$, то их разность есть псевдохарактер на конечной группе $G / G_{0}$, т. е. нулевая функция. Поэтому пространство $\widetilde{H}^{2}(G)$, изоморфное $Q P(G)$, также конечномерно. Теперь утверждение следует из теоремы 1 и из теоремы конечномерности ванэстовских когомологий для групп Ли с конечным числом компонент связности [1, гл. III, следствие 7.3]. 
Благодарности. Автор выражает признательность А. С. Мищенко за постоянное внимание к работе, рецензентам за чрезвычайно полезные замечания, позволившие улучшить изложение, и Ж.-П. Серру за информацию о публикациях Глисона по локальной тривиальности главных расслоений над группами Ли. Бо́льшая часть результатов статьи была получена во время пребывания автора в GMD-SCAI (Sankt-Augustin) в июле и августе 2000 г., и автор благодарит Т. Ленгауэра, Э. Хотцеля и Ф. Шварца за гостеприимство.

\section{ЛИТЕРАТУРА}

1. Гищарде A. Когомологии топологических групп и алгебр Ли. Мир, М., 1984.

2. Фейгин Б. Л., Фукс Д. Б. Когомологии групп и алгебр Ли. Современные проблемы математики. Фундаментальные направления, Т. 21, Итоги науки и техники, ВИНИТИ, M., 1988, 121-209, 215.

3. Хелгасон C. Дифференциальная геометрия и симметрические пространства. Мир, M., 1964.

4. Хьюитm Э., Росс K. А. Абстрактный гармонический анализ. Т. 1, Наука, М., 1975.

5. Штерн А. И. Устойчивость представлений и псевдохарактеры. Доклад на Ломоносовских чтениях, МГУ, М., 1983.

6. Штерн А. И. Непрерывные псевдохарактеры на связных локально компактных группах являются характерами. Функц. анализ и его прил., 27, вып. 4, 94-96 (1993).

7. Besson G. Séminaire Cohomologie bornée, Éc. Norm. Sup. Lyon, Report, Février 1988.

8. Gromov M. Volume and bounded cohomology, Inst. Hautes Études Sci. Publ. Math., 56 (1982), 5-99 (1983).

9. Guichardet A., Wigner D. Sur la cohomologie réelle des groupes de Lie simples réels. Ann. Sci. Éc. Norm. Sup. 11, 277-292 (1978).

10. Iwasawa K. On some types of topological groups. Ann. of Math., 50, 507-558 (1949).

11. Lee D. H. Supplements for the identity component in locally compact groups, Math. Z., 104, 28-49 (1968).

12. Palais $R$. S. On the existence of slices for actions of non-compact Lie groups. Ann. of Math. (2), 73, No. 2, 295-323 (1961).

13. Paterson A. L. T. Amenability. Amer. Math. Soc., Providence, R.I., 1988.

14. Segal G. Cohomology of topological groups. Symposia Math., Vol. IV, Acad. Press, London, 1970, pp. 377-387.

15. Serre J.-P. Trivialité des espaces fibrés. Applications. C. R. Acad. Sci. Paris, 230, 916-918 (1950).

16. Serre J.-P. Extensions des groupes localement compacts (mimeographed notes). Bourbaki seminar lecture on March 27, 1950.

17. Shtern A. I. Remarks on Pseudocharacters and the Real Continuous Bounded Cohomology of Connected Locally Compact Groups. Sfb 288 Preprint No. 289, Humboldt University, Berlin, 1997.

18. Shtern A. I. A criterion for the second real continuous bounded cohomology of a locally compact group to be finite-dimensional. Acta Appl. Math. (в печати).

19. Shtern A. I. Bounded continuous real 2 -cocycles on simply connected simple Lie groups and their applications. Russian J. Math. Phys., 8, No. 1, 122-133 (2001).

20. Shtern A. I. Remarks on pseudocharacters, and the real continuous bounded cohomology of connected locally compact groups. Ann. Global Anal. Geom. (в печати)

21. Terp $C$. On locally compact groups whose set of compact subgroups is inductive. Seminar Sophus Lie [J. Lie Theory], 1, 73-80 (1991). 